\title{
Comment on "Unexpected reduction of rf spin resonance strength for stored deuteron beams"
}

\author{
S. R. Mane \\ Convergent Computing Inc., P.O. Box 561, Shoreham, New York 11786, USA
}

(Received 17 March 2008; published 4 June 2008)

\begin{abstract}
I point out that the claim of an "unexpected" reduction of the resonance width in spin-flip studies reported in the paper by Krisch et al. [Phys. Rev. ST Accel. Beams 10, 071001 (2007)] is invalid, because the authors employed a theoretical expression for the resonance width which did not account for all of the contributions to the spin flip induced by a radial field rf dipole spin flipper.
\end{abstract}

PACS numbers: 29.27.Bd, 29.27.Hj, 41.75.Ak

In a recent paper, Krisch et al. [1] have reported an "unexpected reduction" of the spin-flip resonance width for a stored polarized deuteron beam, via studies performed using a radial field rf dipole spin flipper. In this Comment, I point out that the claim of an unexpected result in the above paper is invalid, because the authors employed a theoretical expression for the resonance width which did not account for all of the contributions to the spin flip induced by an rf dipole. I present the theoretical expressions for the spin-flip resonance width for an rf solenoid and rf dipole below, and discuss them in more detail later in this paper. For a particle of charge $e$, mass $m$, velocity $\boldsymbol{v}$, momentum $p$ (and Lorentz factor $\gamma=1 / \sqrt{1-v^{2} / c^{2}}$ ), and magnetic moment anomaly $G=(g-2) / 2$, the spinflip resonance strength induced by an $\mathrm{rf}$ solenoid is

$$
\varepsilon_{\mathrm{sol}}=\frac{G+1}{\pi 2 \sqrt{2}} \frac{e}{p c}\left(\int B_{\mathrm{sol}} d \ell\right)_{\mathrm{rms}} .
$$

The last factor is the rms integrated field on the rf solenoid. For an rf dipole the expression for the spin-flip resonance strength is

$$
\varepsilon_{\mathrm{dip}}=\frac{G \gamma+1}{\pi 2 \sqrt{2}} \frac{e}{p c}\left(\int B_{\mathrm{dip}} d \ell\right)_{\mathrm{rms}}+I_{\beta_{v}} .
$$

The first term is analogous to that for an $\mathrm{rf}$ solenoid. However, there is a second contribution $I_{\beta_{v}}$ to the resonance strength, because an rf dipole also induces coherent vertical betatron oscillations, which in turn couple to the spin and modify the resonance strength. I shall present a more detailed expression for $\varepsilon_{\text {dip }}$ as an integral over the ring optical functions below. It will be seen that the resonance strength $\varepsilon_{\text {dip }}$ depends on the vertical betatron tune. The fact that the resonance strength depends on the vertical betatron oscillations (for an rf dipole) was noted already in 1963 by Simonyan and Orlov [2]. See also formulas by Kondratenko [3]. Bai et al. [4] used an rf dipole to adiabatically induce a large amplitude coherent betatron oscillation to successfully achieve adiabatic spin flip without emittance blowup, when crossing an intrinsic spin resonance at the AGS. In practice, the entrance and exit fringe fields of an $\mathrm{rf}$ solenoid can also excite betatron oscillations in the ring, and these can couple to the spin. This is a higher order effect and is not included in Eq. (1), but it can be noticeable, for example, if the vertical betatron frequency is close to an integer multiple of the beam circulation frequency plus or minus the rf solenoid frequency.

The authors in [1] neglected the contribution of the coherent vertical betatron oscillations to $\varepsilon_{\text {dip }}$, and stated the resonance strength as only ("SC" for SPIN@COSY)

$$
\varepsilon_{\mathrm{sc}}=\frac{G \gamma+1}{\pi 2 \sqrt{2}} \frac{e}{p c}\left(\int B_{\mathrm{dip}} d \ell\right)_{\mathrm{rms}},
$$

which omits an essential contribution to the resonance width. Towards the end of their paper [1], the authors stated that "Two independent approaches...now challenge the derivation of Eq. (5) [which is Eq. (3) above, in different notation]; they suggest that its factor $(1+G \gamma)$ should instead be proportional to $G \gamma$." The authors then compared the following modified expression to their data:

$$
\varepsilon_{\mathrm{sc}}^{\prime}=\frac{G \gamma}{\pi 2 \sqrt{2}} \frac{e}{p c}\left(\int B_{\mathrm{dip}} d \ell\right)_{\mathrm{rms}} .
$$

However, such a modification does not contain a dependence on the vertical betatron tune, and is incompatible with the experimentally known behavior of radial field rf dipole spin flippers. In fact, the authors actually displayed a graph of the variation of the resonance strength as a function of the vertical betatron tune, in a sweep of the vertical betatron tune from 3.6 to 3.85 , with a resonance peak at 3.8 (see Fig. 4 in [1]). The data in [1] is therefore inconsistent with both Eqs. (3) and (4).

Note that the expression in Eq. (3) was also employed in previous spin-flip studies by the SPIN@COSY collaboration. For example, the paper [5] contains a compilation of resonance widths from spin-flip studies at several rings (IUCF Cooler, MIT-Bates South Hall Ring, and COSY), and the theoretical results therein also disagreed with the data. The data listed in [5] included spin-flip studies using both rf solenoids and rf dipoles, for both stored proton and deuteron beams (including vector and tensor polarization for the latter). Note that the theoretical expressions for the resonance strengths in [5] contained a factor of 2 error (for both rf solenoids and dipoles); this was corrected by Bai, MacKay, and Roser [6]. Note in addition that the compi- 
lation of data in [5] included also spin-flip studies using a nearly full Siberian Snake [7-9]. The theoretical expressions for the resonance strengths in Eqs. (1) and (2) require modifications if the ring contains a nearly full Siberian Snake. However, I shall not treat rings with Siberian Snakes in this paper.

I now present an expression for $\varepsilon_{\text {dip }}$ as an integral over the ring optical functions, for a planar ring without horizontal-vertical betatron coupling. Let the independent variable be the generalized azimuth $\theta$, and define $f^{\prime}=$ $d f / d \theta$ for any function $f$. Let the local radius of curvature of the reference orbit be $\rho$ and $R$ be the average ring radius. Denote the magnetic rigidity by $B \rho$ and the vertical betatron tune by $\nu_{z}$. For a planar ring, the spin tune is $\nu=G \gamma$. Let us denote the coherent vertical betatron by $z_{\text {coh }}$, and its resonant part by $z_{\text {res }}$. The concept of the "resonant part" of a coherent vertical betatron oscillation can be illustrated by solving the simple example of a smooth focusing model. Let $B_{\mathrm{rms}} L$ and $\nu_{\mathrm{rf}}$ be, respectively, the rms field integral and tune of the rf dipole, $\chi$ be an initial phase, and $\theta_{\mathrm{rf}}$ be the azimuthal location of the rf dipole. For a smooth focusing model, Hill's equation with a driving term for the vertical oscillations is

$$
\begin{aligned}
z^{\prime \prime}+\nu_{z}^{2} z= & -R \frac{\sqrt{2} B_{\mathrm{rms}} L}{B \rho} \cos \left(\nu_{\mathrm{rf}} \theta+\chi\right) \\
& \times \sum_{j=-\infty}^{\infty} \delta\left(\theta-\theta_{\mathrm{rf}}-2 j \pi\right) .
\end{aligned}
$$

Then the coherent betatron oscillation $z_{\mathrm{coh}}$ is given by

$$
\begin{aligned}
z_{\mathrm{coh}}= & -\frac{R}{\pi 2 \sqrt{2}} \frac{B_{\mathrm{rms}} L}{B \rho} \sum_{k=-\infty}^{\infty}\left\{\frac{e^{i\left[\left(k+\nu_{\mathrm{rf}}\right) \theta+\chi-k \theta_{\mathrm{rf}}\right]}}{\nu_{z}^{2}-\left(k+\nu_{\mathrm{rf}}\right)^{2}}\right. \\
& \left.+\frac{e^{i\left[\left(k-\nu_{\mathrm{rf}}\right) \theta-\chi-k \theta_{\mathrm{rf}}\right]}}{\nu_{z}^{2}-\left(k-\nu_{\mathrm{rf}}\right)^{2}}\right\} .
\end{aligned}
$$

Next suppose that $G \gamma=n+\nu_{\mathrm{rf}}$. Then the resonant term $z_{\text {res }}$ will be given by $k=-n$ (in the second sum).

Treating the general case, the term $I_{\beta_{v}}$ can interfere either constructively or destructively with the direct coupling of an $\mathrm{rf}$ dipole to the spin: the overall resonance strength may be decreased by the contribution of the coherent vertical betatron oscillations. Then $[2,3]$

$$
\begin{aligned}
\varepsilon_{\mathrm{dip}}= & -\frac{i}{2 \pi} \oint\left[\left(G^{2} \gamma^{2}+G\right) \frac{z_{\text {res }}^{\prime}}{\rho}+(1+G)\right. \\
& \left.\times\left(\frac{1}{\rho}\right)^{\prime} z_{\text {res }}\right] e^{i G \gamma \Theta} d \theta,
\end{aligned}
$$

where

$$
\Theta=\int_{0}^{\theta} \frac{R}{\rho(\tilde{\theta})} d \tilde{\theta}
$$

By using the following result

$$
\oint \frac{d}{d \theta}\left[\frac{z_{\mathrm{res}}}{\rho} e^{i G \gamma \Theta}\right] d \theta=0,
$$

we can eliminate $z_{\text {res }}^{\prime}$ to obtain the alternative expression

$$
\begin{aligned}
\varepsilon_{\mathrm{dip}}= & \frac{1}{2 \pi} \oint\left[i\left(G^{2} \gamma^{2}-1\right)\left(\frac{1}{\rho}\right)^{\prime} z_{\mathrm{res}}\right. \\
& \left.-\left(G^{2} \gamma^{2}+G\right) G \gamma \frac{z_{\mathrm{res}} R}{\rho^{2}}\right] e^{i G \gamma \Theta} d \theta .
\end{aligned}
$$

Now both terms are proportional to $z_{\text {res }}$; hence their magnitudes are directly comparable. Note that the coefficients of the terms are very different between Eqs. (7) and (10). The essential point is the dependence of the resonance strength on the betatron oscillations, not whether the coefficients are proportional to $(1+G \gamma)$, etc. Note also that Eqs. (7) and (10) give the whole resonance strength; there is no separation into a "direct" spin coupling and betatron oscillations.

For a smooth focusing model, where $\rho=R$ is constant, $(1 / \rho)^{\prime}=0$ and $\Theta=\theta$, and dropping a global phase factor, the resonance strength is

$$
\varepsilon_{\text {smooth }}=-\frac{1}{\pi 2 \sqrt{2}} \frac{B_{\mathrm{rms}} L}{B \rho} \frac{\left(G^{2} \gamma^{2}+G\right) G \gamma}{G^{2} \gamma^{2}-\nu_{z}^{2}} .
$$

(The solution for the resonance strength for a smooth focusing model was first derived by Kondratenko in 1982 [3].) In the limit $G \gamma \rightarrow 0$, we see that $\varepsilon_{\text {smooth }} \rightarrow 0$. By comparison, Eq. (3) does not vanish as $G \gamma \rightarrow 0$. Although the COSY ring is not a smooth focusing model, for deuterons $G_{d} \simeq-0.142987$ is a small number (and $G_{d} \gamma \simeq-0.20084$ in the studies reported in [1]) and the smooth focusing model helps to motivate that the spin-flip resonance width (for $\nu_{z}=3.6$ ) observed in [1] is smaller than the expression in Eq. (3). The expression in Eq. (11) displays a divergence at $G \gamma=\nu_{z}$, just as the experimental data in [1] display a divergence at $G \gamma=\nu_{z}-4$ [but the expression in Eq. (3) and the modification in Eq. (4) do not depend on $\nu_{z}$ ]. In the opposite limit $|G \gamma| \rightarrow \infty$ (and also $|G \gamma| \gg \nu_{z}$ ) we obtain $\varepsilon_{\text {smooth }} \propto G \gamma$ so that the resonance strength then scales like $|G \gamma|$. In this case we find that the expressions in Eqs. (3) and (11) both approach the same limit.

If there are multiple $\mathrm{rf}$ dipoles in the ring then one must include all the driving terms, taking into account their relative phases and amplitudes. Such a situation would apply to the proposed new RHIC rf dipole spin flipper [10], which contains two rf dipoles in series (for each RHIC ring), as well as two full strength Siberian Snakes in each RHIC ring.

[1] A.D. Krisch et al., Phys. Rev. ST Accel. Beams 10, 071001 (2007).

[2] Kh. A. Simonyan and Yu. F. Orlov, Zh. Eksp. Teor. Fiz. 45, 173 (1963) [Sov. Phys. JETP 18, 123 (1964)]. 
[3] A. M. Kondratenko, Novosibirsk Report No. 82-28, 1982.

[4] M. Bai et al., Phys. Rev. E 56, 6002 (1997).

[5] M. A. Leonova et al., Phys. Rev. ST Accel. Beams 9, 051001 (2006).

[6] M. Bai, W. W. MacKay, and T. Roser, Phys. Rev. ST Accel. Beams 8, 099001 (2005).

[7] B. B. Blinov et al., Phys. Rev. Lett. 81, 2906 (1998).
[8] B. B. Blinov et al., Phys. Rev. ST Accel. Beams 3, 104001 (2000).

[9] V.S. Morozov et al., Phys. Rev. ST Accel. Beams 4, 104002 (2001).

[10] M. Bai et al., Proceedings of the 2007 Particle Accelerator Conference (IEEE, Piscataway, 2007), pp. 1847-1849. 